\title{
Quantum probability: an historical survey ${ }^{1}$
}

\author{
LUigi ACCARDI ${ }^{2}$
}

\section{What is quantum probability}

Quantum Probability (QP) is a new branch of mathematics interconnecting classical probability, functional analysis, pure algebra, quantum physics and information and communication engineering.

The mid seventies is the period that marks the beginning of QP as an autonomous discipline. Since then this cross disciplinary nature has accompanied the development of QP and still now it is one of its points of strength, making it an original new trend in contemporary mathematics as well as one of the earliest pioneers of non-commutative mathematics: a field now flourishing with the more recent development of quantum groups, non-commutative geometry, quantum computer, ...

The developments in this area are taking place at such an high pace and such a broad horizon to make impossible any attempt of a detailed synthesis in a single paper. In what follows I shall give a quick historical survey of QP pointing out some of the main problems which have driven these developments and of the main applications to quantum physics.

\section{The early period}

Several notions, tools and single results, now stably entered in the domain of QP, were developed simultaneously with the birth of quantum mechanics. However these developments occurred independently one another, in several different fields such as functional analysis, the foundations of quantum theory,

\footnotetext{
${ }^{1}$ Abstract of an invited talk to the Annual meeting of the Japan Mathematical Society , Tokyo 26 March 1999; to appear as invited contribution in: Proceedings of the Conference on Probability on Algebraic Structures, March 24, 1999, G. Budzban, P. Feinsilver, and A. Mukherjea (eds.), American Mathematical Society, Contemporary Mathematics (CONM) book series

${ }^{2}$ Graduate School of Polymathematics, Nagoya University and Centro Vito Volterra, Università di Roma "Tor Vergata".
} 
signal processing, quantum optics, operator algebras, quantum field theory, ... and the design to build QP as a unifyed discipline was absent in these early papers.

Important probabilistic ideas are present in the work of practically all the main contributors to quantum theory, in particular Heisenberg, Born, Jordan and, later on, Feynman but, from the point of view of mathematical formalization, the algebraic approach to probability theory can be traced back to von Neumann's classical monograph [voN32]. Even if von Neumann never used or precisely defined the term algebraic probability space, this notion begun to emerge in his monograph as a natural linguistic framework to unify classical and quantum probability and the environment for this unification was first identified by him in the theory of Hilbert spaces and later enlarged into the theory of operator algebras. In particular the following ideas, now generally accepted, were present, in a more or less implicit form, in von Neumann's monograph (cf. [AcOb99] for an exposition in contemporary language):

- a *-algebra of operators on some Hilbert space is the quantum analogue of the measurable functions on a probability space. Here and in the following, unless the contrary is explicitly stated, algebra means associative algebra with identity over the complex numbers.

- a state (positive linear functional with $\varphi(1)=1$ ) on this algebra is the analogue of the integral with respect to a probability measure. In particular the trace, on the bounded operators on a Hilbert space, plays the role of the Lebesgue measure on $\mathbf{R}$ or of the counting measure on $\mathbf{N}$

- a self-adjoint operator, or observable, is the analogue of a random variable (so random variables are embedded into a non-commutative algebra)

- for non-commuting observables on a Hilbert space $\mathcal{H}$ there is no natural way to define a joint probability distribution with respect to an arbitrarily given quantum state inside the same Hilbert space.

von Neumann's goal was to describe the mathematical formalism used in quantum theory and no attempt was made in his monograph to deduce it.

After the publication of his monograph, von Neumann devoted much efforts to the generalization of the quantum formalism and the results of these efforts were, from one side the birth of the theory of operator algebras, now an established mathematical discipline, and from the other side the birth of continuous geometry and quantum logics, both generalizations of classical projective geometry. Inspired by Birkhoff and von Neumann's quantum 
logics, Mackey made the first mathematical attempt to deduce the quantum formalism. He listed a set of plausible axioms [Ma76], which should be satisfied by any statistical theory, and deduced from these axioms that to any such theory one can associate an orthocomplemented lattice (or quantum logic), whose elements represent the events. In Mackey's scheme quantum theory was characterized, among the statistical theories, by the property that its lattice of events is isomorphic to the lattice of the closed sub-spaces of a Hilbert space. The attempts to justify this property in terms of plausible physical assumptions, led to a large literature on quantum logics which, even if not completely successful in its main goal, i.e. to explain the conceptual and physical origins of the quantum formalism, produced two interesting mathematical results:

(i) an infinite dimensional extension of the coordinatization theorem of projective geometry [Pir76]

(i) Gleason's theorem (for a statement and an elementary proof of Gleason's theorem cf. Cooke and Keane's article in [QPII] or [Par92]). The long standing problem of extending Gleasons's theorem to an arbitrary von Neumann algebra, was solved by Paskiewicz [Pas85] (cf. Kruszynski's survey in [QPI] for an exposition of the basic ideas of the proof).

On the physical front, the program to extend the standard quantum theory in order to include a description of quantum dissipative phenomena begun to emerge from two different motivations:

i) the attempt to deduce quantum kinetic equations (Pauli, van Hove), which lead to the master equations.

ii) The attempts to describe the phenomenon of signal damping in quantum optics and quantum communication theory, which led to the introduction, by Lax, of quantum white noise and quantum Brownian motion (cf. [Ac90] for an historical survey).

Both these lines of research will find their natural formulation and solution in the stochastic limit of quantum theory (see below).

\section{The 70's}

During the late Sixties and early Seventies the rigorous theory of equilibrium classical statistical mechanics on lattice systems, originated in the 
pioneering works of van Hove and Bogolyubov, was developed by Dobrushin, Minlos, Ruelle, ... and the problem of extending these achievements to quantum spin systems naturally arose and was first considered by Araki and others. Moreover methods of classical probability were introduced in the theory of bosonic fields through the pioneering works of Symanzik and Nelson and the hope to obtain similar results for fermionic fields naturally lead to the problem of extending these probabilistic notions to the non commutative domain.

Because of these problems it became urgent to fill the gaps left open by von Neumann's probabilistic scheme, namely to develop the non commutative analogues of the notions of:

- stochastic process, in particular Markov processes

- conditional expectation,

- statistical dependence, in particular Markovianity

- Markovian semigroups

- statistical independence

- central limit theorems

Without these notions the development of a new probability theory could not go beyond some rather superficial analogies.

In the period between 1973 and 1980 the solution of these problems begun to emerge from the joint and often independent work of several researchers.

It is not easy to separate the historical developments of these problems because their histories, although different, are continuously intersecting. The first four of these problems are strictly connected. In fact the notion of Markov process presupposes, for its formulation as well as for the explicit construction of quantum Markov chains, that of stochastic process and of conditional expectation and in its turn should imply a natural notion of Markov semigroup.

The general notion of algebraic stochastic process was introduced in [Ac74], [Ac75] and further generalized and completed with a reconstruction theorem in [AFL82]. The problem to give a general formulation of the Markov property, to deduce from it the construction of non trivial examples of quantum Markov process (chains in the discrete case) and to prove that they are 
canonically associated to quantum Markovian semigroups (irreversible generalizations of the Schrödinger evolution), was solved in [Ac74]. The papers [Ac76], [Ac78a] studied, and solved in some particular cases, the converse problem, later became known under the name of dilation problem, i.e.: given a quantum Markovian semigroup which additional information do we need to construct a quantum Markov process such that the Markovian semigroup, canonically associated to it, is the given one? In the classical case only the initial distribution is needed. In the quantum case this is true only at the cost of obtaining a weak (i.e. non conservative) process [BhP95].

The structure of the generators of a quantum Markovian semigroup was clarified in the finite dimensional case [GKS76] and, slightly later, in the norm bounded case [Li76].

From the mathematical point of view the most interesting new feature emerging from the construction of quantum Markov chains was the necessity to generalize the notion of quantum conditional expectation introduced by I. Segal in the case of a trace and in a general $C^{*}$-algebra by Umegaki [Ume54]. A Umegaki conditional expectation on $C^{*}$-algebra is a norm one projection onto a sub- $C^{*}$-algebra. This notion is very important in many applications to operator algebras and quantum probability. However, for the purposes of quantum probability, in particular for the construction of quantum Markov chains, it is too restrictive and in some cases only product states are compatible with such conditional expectations. This is at odd with the classical case and quite unsatisfactory from a probabilistic point of view because the role of conditioning is precisely that of describing statistical dependences, i.e. deviations of a state from a product one. For these reasons a notion of generalized conditional expectation was needed in which the projection property was lost. This is the mathematical counterpart of the main difference between classical and quantum physics: in classical physics a measurement does not disturb the system on which it is done and therefore to perform twice the same measurement of a discrete observable in an very short time interval is equivalent to perform a single measurement (projection property). In quantum physics things go otherwise.

In [Ac74] only type I von Neumann algebras were considered. The solution of the quantum conditioning problem in the general case had to wait for some years [AcCe82] and had immediate implications not only in quantum probability but also on the theory of operator algebras [Pe84], [Pe88] and in algebraic quantum field theory. For example, the canonical conditional expectation, introduced in this paper, played a crucial role in the solution 
of the Stone-Weierstrass conjecture and in the theory of standard split $\mathrm{W}^{*}$ inclusions [Lo84].

When a Umegaki conditional expectation exists, the theory of quantum Markov processes becomes just a rephrasing of the classical one and was described in $[\mathrm{Ac} 78 \mathrm{~b}]$. Although less exciting from a theoretical point of view, this subclass of quantum Markov processes, the expected processes, is important because almost all the examples of quantum Markov processes in continuous time considered up to now belong to this class.

The first general and purely algebraic quantum central limit theorems, under the assumption of boson or fermion statistical independence, were obtained by von Waldenfels and Giri [GivW78], [vWa78] who also discovered a surprising connection between these results and the canonical commutation and anti-commutation relations of quantum mechanics. These results initiated the algebraic investigation of the notion of statistical independence which lead to several algebraic generalizations of this notion far beyond the original notions of bosonic and fermionic independence (cf. [Schü93] for a survey). This line of research will find a new vigor and enrichment in the 90's (cf. below).

In these years, the experiments of Aspect et al. on the verification of Bell's inequalities, contributed to a revival of interest on the foundational and interpretational problems of quantum theory, in particular the problem of hidden variables. This problem has many mathematically inequivalent formulations in the physical literature, but most of them have in common the attempt to construct a classical probabilistic model for quantum theory and therefore it also contributed to focus the attention of researchers on the role of probability in quantum theory. The notion of statistical invariant [Ac81a], [AcFe82] was an outcome of this debate.

This notion provided the first intrinsic, i.e. model independent, distinction between classical and quantum probability just as the analogue notion of geometrical invariant allows the same distinction between classical and non euclidean geometry.

The name statistical invariant also underlines the difference between this notion and the old von Neumann result about non existence of joint probabilities inside the original Hilbert space. von Neumann's result is model dependent because by enlarging the Hilbert space one can construct uncountably many joint probabilities. On the contrary, as a corollary of the theory of statistical invariants, one can prove that there are sets of experimentally measurable statistical data (conditional probabilities, correlations, 
...) which cannot be deduced from a single set of joint probabilities (Kolmogorov model). With this mathematical result the problem of deciding between the classical and the quantum probabilistic model is reduced to an experimental one. In particular some inequalities satisifed by some statistical data, related to experiments in quantum theory, such as the two slit type experiments (Heisenberg-Feynman) or the Einstein-Podolsky-Rosen type experiments (Bell [Bll64]), which were at the origin of an interesting and still active debate about the foundations of quantum theory acquire a simple mathematical interpretation as necessary conditions for the existence of a Kolmogorov probabilistic model for these statistical data. For a survey of this debate cf. the book [Ac97].

The experimental violation of these inequalities [AspDR82] can thus be interpreted as the experimental proof that the framework of classical probability is too narrow for the needs of contemporary physics and a more general and powerful formalism is needed. The goal of quantum probability is to develop such a formalism.

In this spirit one could say that, as relativity reduces the structure of the laws of space to a physical problem, quantum probability does the same for the laws of chance.

\section{The 80's}

The basic results in this decade concern the following topics:

- Quantum stochastic calculus

- Construction of quantum Markov processes through quantum stochastic calculus

- Quantum filtering and prediction

- Deduction of the mathematical model of classical and quantum probability from physically meaningful axioms

- Quantum conditioning: general case

- Quantum information theory and quantum entropy

- The stochastic limit

- Free independence 
The problem of constructing quantum Markov processes was reduced, through the quantum Feynman-Kac formula [Ac78b], to the construction of unitary Markovian cocycles. However the techniques used to this goal were still relying on standard operator theoretical methods.

The situation changed with the introduction of quantum stochastic calculus by Hudson and Parthasarathy [HuPa84] and their proof of the fact that this is a new, purely quantum probabilistic, technique allowing to construct unitary Markovian cocycles and therefore quantum Markov processes. The quantum Brownian motion, at the basis of the new calculus, was already known in the physical literature, but the quantum Poisson process was a new contribution of these authors and provides an important new mathematical tool for the description of physical systems.

The generality and flexibility of the new method, its potential applications to physics, its connections with classical probability, motivated an intense activity so that the overwhelming fraction of papers produced in this decade turns out to be in one way or another related to quantum stochastic calculus.

A synthesis of the mathematical aspects of stochastic calculus before the late 80's is contained in the monograph [Par92] and additional material on specific topics can be found in the QP series, in the series "Seminaire de Probabilites", Springer LNM, starting from 1984/85 as well as in Meyer's monograph [Mey93]. In fact, through the effective championing of P.A. Meyer, the ideas of quantum stochastic calculus and some ideas of QP begun to spread among classical probabilists.

Quantum stochastic calculus is also a basic ingredient of another important achievement of this decade: Belavkin's quantum filtering and prediction theory [Bel85]. This allows to exploit the whole information of the stochastic process contained in the unitary Markovian cocycle, solution of a SDE, in contrast to the partial information contained in the generator of the Markovian semigroup (master equation). In concrete physical models, for example atoms interacting with a radiation field, this makes a big difference because the latter only allows to estimate decay times and energy shifts in atomic levels, while the former also includes measurable informations on field quantities such as intensity or photon counting statistics [Bar86]. Nowadays the basic ingredients of quantum filtering are, more or less explicitly, present in all models of quantum measurement based on classical or quantum stochastic calculus. A characterization of the flows that can be obtained by the filtering procedure was obtained in [Bel97].

A characterization of the generators of general strongly continuous Marko- 
vian semigroups, is still missing. The best presently available results, which cover several cases of importance for the applications, are due Chebotarev and Fagnola, who derived criteria of existence, uniqueness, unitarity of solutions for classical and quantum generators [CheFa93], based on some inequalities which in many important cases can be directly verified.

A necessary step to make quantum stochastic calculus an applicable tool for quantum physics was to explain the dynamical origins of the quantum Brownian motion and of the quantum Poisson process. In other words, the definition of these processes involves several quantities such as the variance, the state space and, in the case of Poisson process, the choice of a unitary operator. The problem was to deduce the structure of these quantities from the basic Hamiltonian laws of quantum theory so to overcome the purely phenomenological level of the models used in the, now numerous, applications of quantum stochastic calculus to physics. This is a typical problem of non equilibrium quantum statistical mechanics.

The solution of this problem was obtained by the stochastic limit of quantum theory, a research program which started from the papers [AcFrLu87], [AcFrLu90] and proceeded through an increasingly complex series of physical models. The main result of these papers was to show the mechanism through which quantum stochastic differential equations arise as limits of usual Schrödinger equations. The problem was first solved for quantum diffusions, i.e. processes driven by quantum Brownian motion. The, much more difficult, problem of the determination of the dynamical origins of the quantum Poisson process was solved in [AcLu91a],[AcLu91b].

Also of these years is the solution of another traditional problem on the foundations of quantum theory, namely the deduction of the mathematical model of classical and quantum probability from a set of simple, plausible and physically meaningful axioms [Ac82].

The problems of quantum information theory were related to the notion of relative entropy in von Neumann algebras [OHT81] and led to the notions of compound channel, generalizing the classical technique of coupling of two stochastic processes, and of mutual information [Oh83] with applications to quantum communication processes [OhWa85]. A complete, and pleasant to read, account of quantum entropy is the monograph [OhPe93]: the final part of the volume is particularly recommended for the wealth of intuitions and of possible developments.

In 1983 Voiculescu discovered the notion of free independence [Voic91] (cf. the monograph [VoDyNi92] for a survey) by abstracting the properties of the 
generators of the GNS representation, with respect to the trace, of the group algebra of the free group with $N$ generators and proved the corresponding central limit theorem identifying the limit distribution as the Wigner semicircle law.

Speicher [Sp90] proved that the quantum probabilistic techniques, developed in von Waldenfels original papers to prove the quantum central limit theorems, can be applied without essential modifications to prove several generalizations of Voiculescu's original result. Moreover, taking advantage of the functorial character of the realization of a semi-circle random variable in terms of creation and annihilation operators on the Boltzmannian (full) Fock space, Kümmerer and Speicher introduced the free Brownian motion and the associated notion of free stochastic integrals [KüSp92]. Motivated by this result Fagnola proved, in the note [Fag90], how this notion of stochastic integration can be embedded in the general framework proposed in [AcFaQu90] as an attempt to unify the proliferating notions of stochastic integrals in the same sense as, in classical probability, the general notion of semi-martingale prevents the necessity of developing ex novo a different theory of stochastic integration for each class of semi-martingales.

After its discovery, free independence was realized to describe the statistics of $N \times N$ random matrices in the limit $N \rightarrow \infty$, a problem investigated by Wigner in the 50's in connection with some phenomenological models of nuclear physics and which underwent a revival in the 80's because of its connections with the so-called large $N$ limit in euclidean quantum field theory, in which one introduces a fictitious spin parameter in a real physical model and then studies the approximations of this model when the number of spin dimensions tends to infinity. In particular the logarithmic energy, introduced by Voiculescu [Voic94] as a free analogue of entropy, was shown by Ben Arous and Guionnet [BAGu97] to control the large deviations of the large $N$ limit of Gaussian ensambles. In this direction the best presently known results are due to Petz and Hiai [HiPe98].

Thus Voiculescu's results provide still another example of how ideas and techniques of quantum probability can throw light on deep results of classical probability.

\section{The 90's}

Like most of the developments of QP in the 80's were related in some way to the development of stochastic calculus, much of the developments in 
the 90's were somehow related to the circle of ideas originated in free probability. In particular a multiplicity of free analogues of classical probabilistic results on addition of independent random variable and on the combinatorial relations between moments and cumulants, were established (cf. [Sp98] for a survey).

One important new feature emerged with free independence, with respect to boson, fermion and $q$-deformed independence (with $q \neq 0$ ), that is the role of the non crossing diagrams.

A surprising fact, because not suspected even in the heuristic physical literature, is that precisely these diagrams survive in the stochastic limit of quantum electrodynamics without dipole approximation [AcLu92]. However, due to the strong nonlinearity, the vacuum distribution of the field operator after the stochastic limit (master field, in the field teoretical terminology; quantum noise in the quantum optics terminology) is not the semi-circle law but a nonlinear deformation of it. The attempts to explain this fact lead to the introduction of the notion of interacting Fock space, which is now considered to be the natural candidate to replace the usual Fock space in the case of interacting fields (for a multiplicity of examples supporting this statement cf. [AcLuVo97b]). The fact that the Boson (resp. Fermi) Fock space can be obtained as central limit of some kind of quantum Bernoulli processes was known since the 80's [AcBa87] (resp. [Lu89]). The analogue result for a particular case of interacting Fock spaces, the chronological Fock space, was obtained in by De Giosa and Lu [LuDeG95]. The proof that the vacuum distribution of the field operator was the arcsine distribution was obtained successively by Lu [Lu95], [Lu96] and, independently, by Muraki [Mur96a], [Mur96b]. In [Lu97] it was proved that the class of the vacuum distribution of the field operator in an interacting Fock space may depend on the test function. This is a new phenomenon with respect to what happens in the usual (or $q$-deformed) Fock spaces where only the parameters of this distribution depend on the test function. Recently a new method for the derivation of the above mentioned central limit theorem has been found by Liebscher [Liebs97]. Liebscher's method gives as a bonus the representation of the resulting quantum noise in terms of usual stochastic integrals.

This notion of interacting Fock space is intimately related to another new feature emerged in the stochastic limit of QED: the state space of the combined system atom + master field should be described by a Hilbert module rather than a Hilbert space. This is the mathematical counterpart of the physical phenomenon that, due to the nonlinearity, the degrees of freedom 
of the atom and of the field become entangled after the limit. In the recent physical literature the term entanglement is used simply as synonym of superposition in a tensor product space (cf. however a new, mathematical formulation of this concept, due to Belavkin and Ohya and related to a new notion of quantum entropy introduced by these authors [BeOh99]). What we mean here is different, namely that, even if before the limit atom and field observables commute, after the limit they develop nontrivial commutation relations (in fact an operator generalization of the free commutation relations). In the case of QED Skeide [Ske96] proved that the interacting Fock space structure can be deduced from the Hilbert module structure alone. If this result is a general phenomenon, or if it is specific to the QED Hilbert module, is an open problem.

Several algebraic generalizations of the notion of free independence were studied by Bozejko and Speicher [BoSp91] but neither the statistical meaning of the conditions nor, in most cases, the corresponding central limit distributions were very transparent in these papers also because of the presence of partitions of any order, not only pair partitions, in the expressions of momenta for the limit distributions.

Accardi, Hashimoto and Obata [Aho98] proved that, by considering the GNS representation of free groups, not with respect to the trace but to an arbitrary Haagerup function, one is naturally lead to a new notion of independence, singleton independence, which manifests some new qualitative properties with respect to the usual notions of independence:

(i) the singleton condition is not satisfied

(ii) not only the non crossing pair partitions, but also some singletons give contributions to the limit distribution.

Moreover, using a previous results of Hashimoto [Has97], they identified the limit distributions obtained in this way as the one parameter family of Ullman distributions. Shortly after Accardi and Bozejko [AcBo98] combined Bozejko's continuous fraction convolution with the notion of interacting Fock space and proved that any probability measure on $\mathbf{R}$, with all moments, can be obtained as the vacuum distribution of the field operator in an interacting Fock space so that the combinatorics of the momenta is expressed uniquely in terms of singletons and pair partitions. This phenomenon was called Gaussianization of the given probability measure because in classical probability this combinatorics is characteristic of Gaussian distributions. 
The interest of physicists for the quantum Markov chains increased in the early 1990's with the understanding, by Fannes, Nachtergaele, Werner [FNW92a], [FNW92b], [FNW94], that the valence bond states, introduced and studied by famous theoretical physicists in the attempt of explaining the phenomenon of high temperature superconductivity, are a particular class precisely of those quantum Markov chains which were introduced for purely mathematical reasons in the 70's. In the case of infinite tensor products of matrix algebras (the general definition of quantum Markov chains does not require this restriction) these authors gave an alternative characterization of quantum Markov chains which motivated the name finitely correlated state frequently used to denote this important class of quantum Markov chains. They proved furthermore that the mixing Markov chains corresponding to the valence bond models are pure states. This is rather unexpected in view of the fact that the notion of quantum Markov states was introduced as an analogue of Gibbs measure. A very interesting recent result is due to Matsui [Mat98] who proved that a mixing translation invariant state is a zero energy ground state for a translation invariant nearest neighbor Hamiltonian if and only if it is a pure quantum Markov chain and, if the Hamiltonian satisfies an additional non degeneracy condition, even a finitely correlated state. A nearest neighbor Hamiltonian on a one-dimensional lattice is a formal sum of self-adjoint operators, each of which is localized on two consecutive sites of the lattice (such an Hamiltonian has a rigorous meaning as a derivation on the algebra of quasi-local observables and, by a theorem of Sakai, it generates a quantum dynamics). A zero energy state for such an Hamiltonian is a state giving mean zero to each of these operators separately and not only, as a usual ground state, to their sum.

Another development of these years, in a completely different direction, i.e. the white noise appraoch to classical and quantum stochastic calculus, is likely to be one of the most exciting frontiers of current research both in classical and quantum probability.

This approach too arose from the stochastic limit of quantum theory. The idea is the following: we know that, in the stochastic limit, the Schrödinger equation becomes a stochastic differential equation. On the other hand the formal limit of this equation is a white noise Hamiltonian equation. At this point the conjecture that the two equations coincide is a natural one. Now, using one of the basic principles of the stochastic limit (the time consecutive principle) one can put the white noise equation in normal order. One can prove that this normally ordered equation and the stochastic differential 
equation have the same matrix elements (in the number and the exponential vectors). Since we know that the stochastic equation has a unique unitary solution, it follows that the same is true for the corresponding white noise equation and that the two solutions coincide. Notice that all what said above remains true even in the case of classical stochastic equations. This means that the attempt to develop a stochastic calculus for classical white noise naturally leads to the introduction of quantum probabilistic techniques.

For a full development of a white noise approach to stochastic calculus, an important link was still missing: how to express the Ito formula in white noise terms. This gap was filled in the paper [AcLuVo97a] where the symbolic table

$$
\delta(0) d t=1 \quad ; \quad(\text { normal order }) d t^{2}=0
$$

were shown to play, in white noise stochastic calculus, the same role played, in classical stochastic calculus, by the symbolic formulation of the Ito table

$$
d w^{2}=d t \quad ; \quad d w d t=0
$$

In both cases these symbolic notations acquire a rigorous meaning through limit procedures.

The advantage of the formulation (1) of the Ito table, with respect to the traditional one, is that apparently it does not include an explicit dependence on the process involved (classical or quantum white noise, number process, Poisson noise): in fact this dependence enters in the specification of the corresponding limit relations. This naturally suggests the possibility to use formula (1) also in the computation of formal Ito tables corresponding to higher powers of white noise. This was done in [AcLuVo97a] where it was also shown that already for the square of the white noise the table (1) has to be integrated with a renormalization procedure, thus leading to the notion of renormalized Itô table. The first explicit unitary solution of a singular equation driven by the renormalized square of the white noise was obtained in $[\mathrm{AcVo}$ 97].

In connection with the square of white noise a totally unexpected fact was recently discovered: introducing a renormalization procedure different from the one usually considered in physics, one can construct a Lie algebra canonically associated to the renormalized square of (Boson) white noise and one can prove that this Lie algebra admits a natural representation in an interacting Fock space [ALV99]. It turns out [AcSk99] that this new type of interacting Fock space is canonically isomorphic to the finite difference Fock 
space introduced by Boukas [Bou88], [Bou91] as representation space of an infinite dimensional generalization of the finite difference algebra previously introduced by Feinsilver [Fei87] as a purely mathematical generalization of the Weyl algebra of the Heisenberg commutation relations. Furthermore this algebra is related to a current representation of the Lie algebra of $S L(2, \mathbf{R})$ and this suggests an interesting connection between the square of white noise and the theory of automorphic functions, which is currently under investigation.

The natural framework for the development of a white noise stochastic calculus, as a generalization of both classical and quantum stochastic calculus, is that of Hida distributions [HKPS93], [Ob94] and its recent generalizations [Kuo96]. In this direction several results were obtained by Obata [Ob95], [Ob97a], [Ob97b], [Ob99]. Among them, in particular, the first existence uniqueness and unitarity condition for a first order white noise equation [ChJiOb98] (corresponding to the same result for quantum stochatic differential equations) as well as an intriguing connection between the new distribution spaces which have been recently introduced as generalizations of Hida distributions and the interacting Fock space which was originally introduced for completely different reasons.

\section{Conclusions}

In the present survey, for reasons of space, we have concentrated our attention only on the highlights of the development of QP and a substantial part of results, of interest either for pure mathematics of for their applications to physics, could not even be mentioned. However even such a broad line overview might be helpful to convey to a reader, non expert in the field, the impression of a new branch of mathematics which, in the relatively few years of its existence, has been able to produce relevant and non trivial contributions to the solution of open problems arising in a multiplicity of different fields ranging from the boundary between mathematics and philosophy, e.g. the foundations of probability or the interpretation of quantum theory, to some very technical branches of mathematics such as operator algebras, functional integration, the theory of classical stochastic processes, ..., and arriving to concrete applications to physical problems which were not limited to an elegant rephrasing of things known to physicists, or to the proof of some statement conjectured by them, but have brought to the fore some new effects or phenomena which were not even conjectured in the physical liter- 
ature (the domination of the non-crossing diagrams in the stochastic limit of quantum electrodynamics or of the Anderson model, their re-summation to a unitary operator, the breaking of the standard commutation relations due to nonlinear interactions and the emergence interacting commutation relations, the subsequent emergence of non classical - i.e. neither Bose nor Fermi - statistics, the phenomenon of super-bosonization, ...). The reader interested in these physical applications may look at the book [AcLuVo99b]. A sufficiently faithful photograph of the development of the theory until the early 90 's can be found in the QP series listed below and, for more recent developments, one may refer to the new journal: Infinite dimensional analysis, quantum probability and related topics edited by World Scientific.

\section{Bibliography}

[QP-0] Mathematical problems in the quantum theory of irreversible processes, ed.L.Accardi, V.Gorini, G.Parravicini, Arco Felice 1978.

[QP-I] Quantum probability and applications to the quantum theory of irreversible processes, ed. L.Accardi, V.Gorini, A. Frigerio, Springer LNM n.1055 (1984)

[QP-II] Quantum Probability and applications II , L.Accardi , W.von Waldenfels (eds.) ; Springer LNM N. 1136 (1986)

[QP-III] Quantum Probability and Applications III, Springer LNM N. 1233 (1987)

[QP-IV] Quantum Probability and Applications IV, Springer LNM N. 1396(1989)

[QP-V] Quantum Probability and Applications V, Springer LNM 1442(1990)

[QP-VI] Quantum Probability and Related Topics VI, World Scientific (1991)

[QP-VII] Quantum Probability and Related Topics VII, World Scientific (1992)

[Prob2000] Probability Towards 2000; L. Accardi, Chris Heyde (eds.) Springer LN in Statistics 128 (1998) 
[Ac74] Accardi L.: Noncommutative Markov chains. In: international School of Mathematical Physics, Camerino (1974) 268-295.

[Ac75] Accardi L.: On the noncommutative Markov property (in russian). Funkt. Anal. and its Appl. 9 (1975) 1-8.

[Ac76] Accardi L.: Non relativistic quantum mechanics as a noncommutative Markov process. Adv. in Math. 20 (1976) 329-366.

[Ac78a] Accardi L.: Noncommutative Markov chains with preassigned evolution: an application to the quantum theory measurement. Adv. in Math. 29 (1978) 226-243.

[Ac78b] Accardi L.: On the quantum Feynmann-Kac formula. Rendiconti del seminario Matematico e Fisico, Milano 48(1978) 135-180

[Ac81a] Accardi L.: Topics in Quantum Probability. Physics Reports, 77 (1981) 169-192

[AFL82] Accardi L., Frigerio A., Lewis J.: Quantum stochastic processes Publications of the Research institute for Mathematical Sciences Kyoto University 18 (1982) 97-133.

[AcFe82] Accardi L., Fedullo A.: On the statistical meaning of complex numbers in quantum theory. Lettere al Nuovo Cimento 34 (1982) 161172.

[AcCe82] Accardi L., Cecchini C.: Conditional expectations in Von Neumann algebras and a theorem of Takesaki. Journ. of Funct. Anal. 45 (1982) 245-273

[Ac82] Accardi L.: Some trends and problems in quantum probability. In: Quantum probability and applications to the quantum theory of irreversible processes, ed. L.Accardi et al., Springer LNM n.1055 (1984) $1-19$

[AcBa85] L. Accardi and A. Bach: Quantum central limit theorems for strongly mixing random variables, Z. W. 68 (1985), 393-402.

[AcFrLu87] Accardi L., Frigerio A., Lu Y.G.: On the weak coupling limit problem. in : Quantum Probability and Applications IV Springer LNM N. 1396(1987)20-58 
[Ac90] Accardi L.: Noise and dissipation in quantum theory. Reviews in Math. Phys. 2(1990) 127-176

[AcFaQu90] Accardi L., Fagnola F., Quaegebeur A representation free Quantum Stochastic Calculus Journ. Funct. Anal. 104 (1992) 149-197 Volterra preprint N. 18 (1990)

[AcFrLu90] Accardi L., Frigerio A., Lu Y.G.: The weak coupling limit as a quantum functional central limit, Comm. Math. Phys. 131 (1990) $537-570$

[AcLu91a] Accardi L., Lu Y.G.: The Number Process as Low Density Limit of Hamiltonian Models, Commun. Math. Phys. 637(1991)1-31

[AcLu91b] Accardi L., Lu Y.G.: The low density limit of quantum systems, Jour. Phys. A: 24 (1991) 3483-3512

[AcLu92] Accardi L., Lu Y.G.: The Wigner Semi-circle Law in Quantum Electro Dynamics. Commun. Math. Phys., 180 (1996), 605-632. Volterra preprint N.126 (1992)

[Ac97] Accardi L.: Urne e Camaleonti: Dialogo sulla realtà, le leggi del caso e la teoria quantistica. Il Saggiatore (1997). Japanese translation: Maruzen (1999)

[AcLuVo97a] L. Accardi, Yun Gang Lu, Igor Volovich: Nonlinear extensions of classical and quantum stochastic calculus and essentially infinite dimensional analysis. in: Probability Towards 2000; L. Accardi, Chris Heyde (eds.) Springer LN in Statistics 128 (1998) 1-33

[AcLuVo97b] Accardi L., Lu Y.G., I. Volovich The QED Hilbert module and Interacting Fock spaces. Publications of IIAS (Kyoto) (1997)

[AcVo97] L. Accardi, I.V. Volovich: Quantum white noise with singular non-linear interaction. Volterra preprint N.278 (1997)

[Ac97] Accardi L.: URNE E CAMALEONTI: Dialogo sulla realtà, le leggi del caso e la teoria quantistica. Il Saggiatore (1997). 
[Aho98] Accardi L., Hashimoto Y. and Obata N.: Notions of Independence Related to the Free Group Infinite dimensional analysis, quantum probability and related topics, 1, N. 2 (1998) 201-220 Volterra Preprint (1998) N. 311.

[AcBo98] L. Accardi and M. Bożejko: Interacting Fock spaces and Gaussianization of probability measures, Infinite Dimen. Anal. Quantum Prob. 1 (1998), 663-670. V. Volterra Center Preprint No. 321, 1998.

[AcOb99] Accardi L., Obata N.: Elementary quantum probability (in japanese), Nagoya Mathematical Lectures, vol. 2 (1999)

[AcLuVo99a] L. Accardi, Y.G. Lu, and I.V. Volovich: White noise approach to classical and quantum stochastic calculi, Volterra Preprint, Rome (1999) To appear in the lecture notes of the Volterra International School of the same title, held in Trento. World Scientific (2.000)

[AcLuVo99b] Accardi L., Y.G. Lu, I. Volovich: Quantum Theory and its Stochastic Limit. to be published in the series Texts and monographs in Physics, Springer Verlag (2000); Japanese translation, Tokyo-Springer (2000)

[AcSk99] Luigi Accardi, Michael Skeide: On the Relation of the Square of White Noise and the Finite Difference Algebra, IDA-QP Infinite dimensional analysis, quantum probability and related topics 1 (2.000)

[AspDR82] A. Aspect, J. Dalibard and G. Roger: Experimental test of Bell's inequalities using time-varying analyzers, Phys. Rev. Lett. 49 (1982), 1804-1807.

[Bar86] Barchielli A.: Measurement theory and stochastic differential equations in quantum mechanics. Physical review A 34 (1986) 1642-1649.

[Bel85] Belavkin V.P.: Non-demolition measurement and control in quantum dynamical systems. Proc. of the conf. "Information complexity and control in quantum physics, Udine 1985, Springer-Verlag, (1985) $331-336$.

[Bel97] V. P. Belavkin: Quantum stochastic positive evolutions: characterization, construction, dilation, Commun. Math. Phys. 184 (1997), $533-566$. 
[BeOh99] V. P. Belavkin, M Ohya: private communication

[Bll64] J. S. Bell: On the Einstein Podolsky Rosen paradox, Physics 1 (1964), 195-200.

[BAGu97] G. Ben Arous and A. Guionnet: Large deviations for Wigner's law and Voiculescu's non-commutative entropy, Probab. Theory Related Fields 108 (1997), 517-542.

[BhP95] B.V.R. Bhat and K.R. Parthasarathy, Markov dilations of nonconservative dynamical semigroups and a quantum boundary theory, Ann. Inst. Poincarè 31 (1995) 601-651.

[BoSp91] Bozejko M., Speicher R.: An Example of a Generalized Brownian Motion. Commun. Math. Phys. 137 (1991), 519-531

[Bou88] A. Boukas: Quantum stochastic analysis: a non-Brownian case, $\mathrm{PhD}$ thesis, Southern Illinois University (1988)

[Bou88] A. Boukas: An example of quantum exponential process, Mh. Math., 112 (1991) 209-215

[Bou88] A. Boukas: Stochastic calculus on the finite-difference Fock space, L. Accardi (ed.) Quantum Probability \& Related Topics VI, World Scientific (1991)

[CheFa93] A.M. Chebotarev, F. Fagnola, Sufficient conditions for conservativity of quantum dynamical semigroups. Journal of Functional Analysis, 1993, v.3, N1, 131-153.

[ChJiOb98] D. M. Chung, U. C. Ji and N. Obata Normal-ordered white noise differential equations I: Existence of solutions as Fock space operators Preprint (1998) to appear in "Proc. Italian-Japanese Meeting on Fluctuations and Mathematical Modelling." (1999)

[Fag90] Fagnola F. Quantum stochastic integration with respect to free noises. Preprint Volterra N. 37 (1990) in: Quantum Probability and Related Topics, World Scientific, OP-PQ VI (1991)

[FNW92a] M. Fannes, B. Nachtergaele, and R.F. Werner. Abundance of Translation Invariant Pure States on Quantum Spin Chains. Letters in Mathematical Physics, 25 (1992) $249-258$ 
[FNW92b] M. Fannes, B. Nachtergaele, and R.F. Werner. Finitely Correlated States on Quantum Spin Chains. Commun. Math. Phys., 144 (1992) $443-490$

[[FNW94] M. Fannes, B. Nachtergaele, and R.F. Werner. Finitely Correlated Pure States. J. Funct. Anal., 120 (1994) 511 - 534

[Fei87] P.J. Feinsilver: Discrete analogues of the Heisenberg-Weyl algebra, Mh. Math., 104 (1987) 89-108

[GivW78] von Waldenfels W., Giri N.: An Algebraic Version of the Central Limit Theorem. Z. Wahrscheinlichkeitstheorie verw. Gebiete 42, 129134 (1978).

[GKS76] Gorini V., Kossakowski A., Sudarshan E.C.G.: Completely positive dynamical semigroups of $N$-level systems. Journal of Mathematical Physics 17 (1976) 821-825.

[Has97] Hashimoto Y.: Deformations of the semi-circle law derived from random walks on free groups. Prob. Math. Stat. 18 (1998). V. Volterra Center Preprint 1997

[HKPS93] T. Hida, H.-H. Kuo, J. Potthoff and L. Streit: "White Noise: An Infinite Dimensional Calculus," Kluwer Academic, 1993.

[HiPe98] F. Hiai and D. Petz: Eigenvalue density of the Wishart matrix and large deviations Infinite dimensional analysis, quantum probability and related topics, 1, N. 4 (1998)

[HuPa84] Hudson R.L., Parthasarathy K.R. Quantum Ito's formula and stochastic evolutions. Comm. Math. Phys. 93(1984)301-323

[KüSp92] Kümmerer B., Speicher R.: Stochastic Integration on the Cuntz Algebra $O_{\infty}$. J. Funct. Anal 103 (1992), 372 - 408

[Kuo96] H.-H. Kuo: White Noise Distribution Theory, CRC Press, 1996.

[Liebs97] V. Liebscher: On a Central Limit Theorem for Monotone Noise, Preprint (1997), Infinite dimensional analysis, quantum probability and related topics. N 1 (1999) 
[Li76] Lindblad G. On the generators of quantum dynamical semigroups. Comm. Math. Phys. 48(1976)119-130

[Lo84] Longo R.: Solution of the Stone-Weierstrass conjecture. An application of the theory of standard split $\mathrm{W}^{*}$-inclusions. Invent. Math. 76(1984)145-155

[Lu89] Lu Y.G.: The Boson and Fermion quantum Brownian motions as quantum central limits of quantum Bernoulli processes. Bollettino U.M.I., (7) 6-B (1992), Volterra preprint (1989)

[LuDeG95] De Giosa M., Lu Y.G. : The free creation and annihilation operators as the central limit of quantum Bernoulli process. Preprint Dipartimento di Matematica Universitá di Bari 2 (1995), Random operators and Stoch. Eq. (1997)

[Lu95] Lu Y.G.: The interacting Free Fock Space and the Deformed Wigner Law. Preprint of Centro Volterra (1995), Nagoya J. of Math. vol.145, pp 1-29 (1997).

[Lu96] Lu Y.G.: An interacting free Fock space and the reciprocal Wigner law. Preprint of Centro Volterra (1996), Probability and mathematical statistics, vol. 17, fasc. 1 (1997)

[Lu97] Lu Y.G.: Interacting Fock spaces related to the Anderson model, Infinite dimensional analysis, quantum probability and related topics, 1, N. 2 (1998) 247-283

[Ma76] Mackey G.W. Mathematical foundations of quantum mechanics. Addison-Wesley (1976)

[[Mat98] Taku Matsui: A Characterization of Pure Finitely Correlated States Infinite dimensional analysis, quantum probability and related topics, 1, N. 4 (1998)

[Mey93] P.-A. Meyer: "Quantum Probability for Probabilists," Lect. Notes in Math. Vol. 1538, Springer-Verlag, 1993.

[Mur96a] Muraki N.: A new example of non commutative De MoivreLaplace theorem, in: Quantum stochastic analysis and related fields, N.Obata (ed.), RIMS Kokyuroku 957, Publications of the Research Institute for Mathematical Sciences, Kyoto August (1996) 154-166 
[Mur96b] N. Muraki: A new example of noncommutative "de MoivreLaplace theorem," in "Probability Theory and Mathematical Statistics (S. Watanabe et al, eds.)," pp. 353-362, World Scientific, 1996.

[Ob94] N. Obata: "White Noise Calculus and Fock Space," Lect. Notes in Math. Vol. 1577, Springer-Verlag, 1994.

[Ob95] N. Obata: Generalized quantum stochastic processes on Fock space, Publ. RIMS, Kyoto Univ. 31 (1995), 667-702.

[Ob97a] N. Obata: Integral kernel operators on Fock space - Generalizations and applications to quantum dynamics, Acta Appl. Math. 47 (1997), 49-77.

[Ob97b] N. Obata: Quantum stochastic differential equations in terms of quantum white noise, Nonlinear Analysis, Theory, Methods and Applications 30 (1997), 279-290.

[Ob99] N. Obata: Wick product of white noise operators and quantum stochastic differential equations, to appear in J. Math. Soc. Japan. 51.

[OHT81] Ohya M., Hiai F., Tsukada M.: Sufficiency, KMS condition and relative entropy in von Neumann algebras. Pacific Journal of Mathematics, vol. 96, n. 1, 1981.

[Oh83] Ohya M.: On compound state and mutual information in quantum information theory, IEEE Trans. Information Theory, 29 (1983) 770777 .

[OhWa85] Ohya M., Watanabe N.: Construction and analysis of a mathematical model in quantum communication processes, Scripta Thecnica, Inc., Elect. Commun. Japan., 68, pp. 29-34, 1985

[OhPe93] M. Ohya and Petz: "Quantum Entropy and Its Use," SpringerVerlag, 1993.

[Par92] Parthasarathy K.R.: An Introduction to Quantum Stochastic Calculus Birkhäuser, 1992.

[Pashk85] Pashkiewicz A.: Measures on Projections in $W^{*}-$ Factors. Journal of Functional Analysis 62 (1985) 87-117. 
[Pe84] Petz D.: A dual in von Neumann algebras, Quart. J. Math. Oxford 35 (1984), 475-483.

[Pe88] Petz D.: Conditional expectation in quantum probability, Quantum Probability and Application III, ed. by L. Accardi and W. von Waldenfels Lecture Notes in Math. 1303, Springer, (1988) 251-260.

[Pir76] Piron C.: Foundations of quantum physics. Addison-Wesley 1976

[Schü93] Schürmann, M.: White noise on bialgebras. (Lect. Notes Math., vol. 1544). Berlin Heidelberg New York: Springer 1993

[Ske96] M. Skeide: Hilbert modules in quantum electro dynamics and quantum probability. Comm. Math. Phys. (1998) Volterra preprint N. 257 (1996).

[Sp90] Speicher R.: A new example of "independence" and "white niose". Probab. Th. Rel. Fields, 84 (1990), 141-159.

[Sp98] Speicher,R.: Combinatorial theory of the free product with amalgamation and operator-valued free probability theory. Habilitationsschrift 1994 Memoirs Amer. Math. Soc., No. 627 (1998)

[Voic91] Voiculescu D.: Free noncommutative random variables, random matrices and the $I I_{1}$ factors of free groups. in: Quantum Probability and Related Fields, 473-487, (1991).

[VoDyNi92] D.V. Voiculescu, K.J. Dykema and A. Nica, Free Random Variables, CRM Monograph Ser., Vol. 1, Amer. Math. Soc., 1992.

[Ume54] Umegaki, H. (1954): Conditional expectation in an operator algebra. Tohoku Math. J. 6, 177-181.

[Voic94] D. Voiculescu: The analogues of entropy and of Fisher's information measure in free probability theory, II, Invent. Math. 118 (1994), 411440 .

[voN32] von Neumann J.: Mathematical foundations of quantum mechanics. Princeton University Press 1955, Die Mathematischen Grundlagen der Quantenmechanik, Springer Verlag, 1932. 
[vWa78] von Waldenfels, W.: An algebraic central limit theorem in the anticommuting case. Z. Wahrscheinlichkeitstheorie verw. Gebiete 42, 135-140 (1978). 